\title{
Forward Motility is Essential for Trypanosome Infection In the Tsetse Fly
}

Brice ROTUREAU, Cher-Pheng OOI, Diego HUET, Sylvie PERROT and Philippe BASTIN

Trypanosome Cell Biology Unit, Institut Pasteur \& CNRS, URA 2581,

25, rue du Docteur Roux, 75015 Paris, France

Corresponding author: Brice ROTUREAU; rotureau@pasteur.fr; Trypanosome Cell Biology Unit, Institut Pasteur \& CNRS, URA 2581; 25, rue du Docteur Roux, 75015 Paris, France; Tel 0033140613833 - Fax 0033140613825

Running title: Trypanosome motility is essential in tsetse flies

Key words: Trypanosoma brucei, flagellum, DNAI1, motility, tsetse fly, vector, infection

Number of Figures: 3 (+ 2 Movies) 


\begin{abstract}
African trypanosomes are flagellated protozoan parasites transmitted by the bite of tsetse flies and responsible for sleeping sickness in humans. Their complex development in the tsetse digestive tract requires several differentiation and migration steps that are thought to rely on trypanosome motility. We used a functional approach in vivo to demonstrate that motility impairment prevents trypanosomes from developing in their vector. Deletion of the outer dynein arm component DNAI1 results in strong motility defects but cells remain viable in culture. However, although these mutant trypanosomes could infect the tsetse fly midgut, they were neither able to reach the foregut nor able to differentiate into the next stage, thus failing to complete their parasite cycle. This is the first in vivo demonstration that trypanosome motility is essential for the accomplishment of the parasite cycle.
\end{abstract}




\section{Introduction}

Trypanosoma brucei are protozoan parasites responsible for human African trypanosomiasis or sleeping sickness, a neglected tropical disease in Central Africa (Simarro et al., 2008, Chappuis et al., 2010). They also cause nagana in cattle, a similar disease with major socio-economical consequences (Brun et al., 2009). Trypanosomes proliferate as extra-cellular parasites in the bloodstream of their mammalian host and can cross the bloodbrain barrier to provoke severe neurological symptoms causing death in the absence of treatment (Brun et al., 2009). Trypanosomes are transmitted by the bite of the tsetse flies, which become vectors when they consume a blood meal from an infected mammal. To complete their life cycle, trypanosomes initially present in the posterior midgut of the fly have to reach the tsetse salivary glands in order to transform into infective parasites (Vickerman, 1985, Peacock et al., 2007, Oberle et al., 2010, Rotureau et al., 2012). This is not direct and requires several proliferation, differentiation and migration steps that take place for 2 to 3 weeks in a strictly defined chronological order in five distinct fly tissues (Vickerman, 1985, Van Den Abbeele et al., 1999, Sharma et al., 2009, Oberle et al., 2010).

Trypanosomes found in the mammalian blood circulation are trypomastigote forms, where the basal body of the flagellum, that is linked to the DNA of the single mitochondrion called the kinetoplast (Robinson et al., 1991), is present at the far posterior end of the cell. Several hours after their ingestion by the fly, bloodstream trypomastigotes differentiate into procyclic trypomastigotes and proliferate in the posterior midgut of the fly. From there, part of the procyclic trypomastigote population cross the peritrophic matrix, elongate into mesocyclic trypomastigotes and migrate anteriorly along the tsetse alimentary tract, through the anterior midgut and the proventriculus to reach the foregut (Vickerman, 1985). This first crucial migration step is accompanied by a differentiation of the mesocyclic trypomastigotes into dividing epimastigotes, where the basal body of the flagellum is found in an anterior position relative to the nucleus (Van Den Abbeele et al., 1999, Sharma et al., 2008, Rotureau et al., 2011). All these parasite stages possess a flagellum that could be essential for the completion of the parasite cycle (Rotureau et al., 2011). Since the primary function of the $T$. 
brucei flagellum is for motility, it is reasonable to expect that trypanosome motility is required for parasite development.

Different modes of locomotion have been described in unicellular organisms such as gliding for Toxoplasma tachyzoites in the human gut (Heintzelman, 2006), crawling for the green algae Chlamydomonas (Heintzelman, 2006), amoeboid movements for the ubiquitous parasite of the human intestine Entamoeba (Tavares et al., 2000), passive diffusion for Plasmodium sporozoites in the Anopheles hemolymph (Hillyer et al., 2007), or piggybacking for Leishmania infantum amastigotes transported to the viscera via the mononuclear phagocyte system (Kaye et al., 2011). Trypanosomes are efficient swimmers in liquid culture medium: their mobility results from the beating of their flagellum that is generated by dynein motors distributed along the axoneme (review in (Hill, 2010)). RNAi silencing of the paraflagellar rod protein PFR2 in monomorphic bloodstream trypanosomes intraperitoneally injected in mice caused their rapid clearance (Griffiths et al., 2007). According to the authors, this would be explained by the reduction of parasite motility that was monitored in parallel in

vitro. However, to our knowledge, there is no other evidence of the importance of swimming in trypanosome in vivo, especially in the tsetse fly vector.

We reasoned that the dynein intermediate chain (DNAl1) of the flagellum axoneme could be a good target to test whether forward swimming is essential for the accomplishment of the parasite cycle. Inducible RNAi silencing of DNA/1 expression in cultured trypanosomes led to the loss of outer dynein arms along the axoneme microtubule doublets, resulting in the blockage of the tip-to-base wave in the flagellum (Branche et al., 2006). These parasites were still able to proliferate, albeit with a duplication rate reduced by two times, and they were not able to swim forward anymore but only to move slowly backward (Branche et al., 2006). Similar defects were observed in $L C 1^{R N A i}$ mutants also deprived of outer dynein arms (Baron et al., 2007, Ralston et al., 2011).

Here, we have tested whether motility impairment could prevent trypanosomes from developing in their vector, focusing on the first crucial migration step. We found that inhibition of forward motility prevented long trypomastigote parasites from reaching the foregut and 
surprisingly also from differentiating into epimastigote forms. These results demonstrate for the first time in vivo that trypanosome motility has an active role in parasite migration from the tsetse midgut to the foregut and is therefore essential for the accomplishment of the

parasite cycle in the tsetse fly vector. 


\section{Results}

\section{Production of DNAl1 mutant parasites}

We designed a knockout strategy to replace both alleles of the DNAl1 gene by two distinct antibiotic resistance markers. Blasticidin (BLA) and neomycin (NEO-G418) resistance marker coding sequences flanked by the first $100 \mathrm{bp}$ upstream of the ATG and downstream of the stop codon of DNA/1 were successively integrated into the trypanosome genome of the T. $b$. brucei AnTat1.1 pleomorphic strain (WT) by homologous recombination in the DNAl1 locus (Figure $1 \mathrm{~A}$ and S1). These DNAI1 mutant cells (M) were sub-cloned by limiting dilution and several subclones were used for further experiments (M1 to M6). A pool of mutant cells was also transformed with a rescue copy of the DNA/1 gene, fused to GFP at the N-terminus end and integrated in the PFR intergenic region for a constitutive expression, to be used as an add-back control strain $(A B)$. Correct integration of the different constructs was verified on several subclones of each strain by PCR, using primers (F2 and R2) designed to hybridize outside the first $100 \mathrm{bp}$ of the DNAl1 flanking sequences used for recombination (Figure 1A). The expected resistance markers were detected in all the different clones for each construct (Figure 1B). In addition, a band of a size corresponding to the expected product of the endogenous locus (1,968 bp) was detected in all mutant types and clones (Figure 1B). Sequencing proved that this corresponds to an extra copy of the DNAl1 gene, and it was observed no matter the order of the transformation process (from 5 replicates with 6 subclones, not shown). Nevertheless, the intensity of this band appeared to be reduced in mutant cells compared to the untransformed WT control (Figure 1B). At the mRNA level, a significant decrease in DNAl1 expression was observed by RT-PCR in mutant subclones compared to WT and AB trypanosomes (Figure 1C). In agreement with the presence of an extra-copy of the gene, a comparatively small amount of DNA/1 mRNA was still produced in mutant cells (Figure 1C).

To evaluate the loss of outer dynein arms along the axoneme of these parasites at the ultrastructural level, membrane and soluble fractions of the cells were extracted by detergent 
treatment and the resulting cytoskeletons were analyzed by transmission electron microscopy. This well-established procedure allows a better visualization of dynein arms (Branche et al., 2006). Examination of flagellum axoneme cross-sections revealed that virtually all of the nine microtubules doublets were bearing outer dynein arms in wild type cells (Figure 1D-E). However, one to six outer dynein arms were absent from microtubule doublets in flagellum cross-sections of DNAl1 mutant parasites (Figure 1D-E). The correct number of outer dynein arms in $A B$ parasites was restored, proving that the structural organization of the axoneme was recovered after the introduction of the rescue copy of the gene (Figure 1D-E).

\section{DNAI1 mutants are still viable despite strong motility defects}

Since the expression of DNAI1 was not fully abolished in mutant cells, the resulting motility defects were scrutinized in all strains and subclones. Individual parasites were first observed under the microscope to characterize their movement. Flagella of wild-type parasites were actively beating in waves propagated most of the time from tip to base, rarely from base to tip, and resulting in forward linear movements (Movie S1). In contrast, DNAI1 mutant parasites presented uncoordinated flagellum beating characterized by irregular base to tip waves leading to a slow rotation of the cell on itself, or chaotic cell movements of small amplitude (Movie S1). As expected, the behavior of $A B$ parasites in terms of flagellum beating and swimming pattern was equivalent to that of wild-type cells (Movie S1). Motility of parasite populations was then monitored by in silico tracking analyses. This also revealed strong swimming defects in DNAI1 mutants that were mostly seen tumbling, whereas WT and $A B$ trypanosomes were moving in typical long curvilinear tracks (Figure 2A). DNA1 mutant cells did not swim forward anymore but were occasionally seen swimming backward in the same direction. However, this was rarely sustained, the resulting motility was slower and shorter compared to WT and AB cells, and this did not reflect their principal behavior as demonstrated in Movie S1 and Figure 2A where only about $5 \%$ of the cells presented such a tracking pattern. Reduction of motility rapidly leads parasites to sediment at the bottom of 
culture flasks in liquid medium. We used this property to evidence motility impairment in mutant subclones by measuring the optical density that reflects the turbidity of the culture medium. Whereas wild-type cells were able to maintain a homogeneous distribution in the entire volume of liquid medium, DNAI1 mutant parasites sedimented rapidly (Figure 2B). In contrast, the presence of a rescue copy of the DNAl1 gene was sufficient for the cells to remain suspended in the culture flask (Figure 2B). DNAl1 mutants were viable and still able to proliferate, although their growth rate in culture was reduced by at least three fold compared to WT and AB cells (Figure $2 \mathrm{C}$ ).

In summation, although the expression of DNAl1 was not fully abolished in mutant trypanosomes, a reduction was sufficient to drastically impair motility, a phenotype that is fully recovered by the addition of a rescue copy of the DNAl1 gene. We took advantage of the viability of these mutants and used them for in vivo functional studies in tsetse flies.

\section{DNAI1 mutant parasites are unable to reach the foregut of their vector}

The first crucial migration step in the tsetse fly vector occurs from the posterior part of the midgut to the proventriculus. Thus, we focused our observations on the development and behavior of the long non-dividing mesocyclic trypomastigotes involved in this migration. A total of 746 Glossina morsitans morsitans teneral males were infected by feeding with culture medium containing WT cells, DNAl1 mutant subclones 1 or 2 , or $A B$ parasites. Flies were dissected about 30 days after the infective meal (26 to 34 days), i.e. only after the double of the minimal time necessary to obtain a mature infection. This served to negate any possible developmental bias caused by the slower proliferation rate observed in DNAl1 mutant cells in vitro. The organs of interest were dissected independently for each fly. The posterior and anterior midgut, the proventriculus and foregut were checked for the presence of parasites under the microscope. Similar to our previous studies (Rotureau et al., 2011, Subota et al., 2011, Rotureau et al., 2012), parasites were found in $45 \%$ of the posterior midguts, $11 \%$ of the anterior midguts, and $4 \%$ of the foreguts of flies fed with WT trypanosomes (Figure 3A). In contrast, only $13 \%$ of posterior midguts and $7 \%$ of anterior midguts were invaded by 
parasites in flies fed with DNAI1 mutant parasites and no trypanosomes were detected in the foregut of these flies (Figure 3A). These differences were all the more striking than mean infection rates observed with WT parasites were slightly lower when compared to our previous observations in the lab. This could be due to inter-experiment variations observed in this group. Moreover, parasite densities in the different regions of the digestive tract were estimated by eyes as described previously (Subota et al., 2011) and no difference in parasite density was observed between strains in the same region (not shown). This phenotype was reversed upon expression of the DNAl1 rescue copy in $A B$ parasites that resulted in high infection rates in the anterior midgut and foregut (Figure $3 \mathrm{~A}$ ). These results demonstrate that DNAI1 mutants are unable to complete the first migration step of their parasite cycle in the tsetse fly from the posterior midgut to the foregut, and that this phenotype is related to the expression of DNAI1.

\section{DNAl1 mutant trypomastigotes are unable to differentiate into epimastigotes}

When living parasites extracted from the midgut of infected flies were examined under the microscope, DNAI1 mutant parasites presented uncoordinated flagellum beating characterized by irregular base to tip waves that led to chaotic cell movements, whereas WT and $A B$ trypanosomes were actively beating their flagellum in waves propagated most of the time from tip to base. This resulted in efficient forward linear movements only in the WT and AB strains (Movie S2). These observations confirm that the DNAI1 mutant motility phenotype was maintained in midgut trypomastigotes, and demonstrate that despite this impairment some DNAI1 mutant parasites could yet progress in the direction of the proventriculus.

The most striking difference between the DNAI1 mutants and the WT and AB cells was observed when the cells extracted from the anterior midgut were typed according to their general morphology and positions of their nucleus and kinetoplast in relation to one another along the anterior-posterior axis of the cell (Fig.3B). The quantification of cell population by stage revealed that whereas $19 \%$ of control parasites had differentiated into epimastigotes, all DNAI1 mutant trypanosomes remained in the trypomastigote morphotype (Figure 3B-C). 
Here too, this phenotype was complemented in parasites bearing a rescue copy of the DNAl1 gene, among which $7 \%$ of trypanosomes were in the epimastigote stage (Figure 3B- 


\section{Discussion}

Motility was proposed to play a crucial role during the early development of trypanosomes in the tsetse fly, especially during their first migration step from the posterior midgut to the foregut (Van Den Abbeele et al., 1999). Trypanosome migration could be passive as it is the case for Plasmodium sporozoites bathing in the Anopheles hemolymph that passively reach the salivary glands after their egress from the oocytes (Hillyer et al., 2007). However, there is no evidence for the presence of trypanosomes in the tsetse hemolymph, and it seems unlikely that $30 \mu \mathrm{m}$ long trypanosomes could passively diffuse along a digestive tract more than $5 \mathrm{~cm}$ in length, against the active forces of digestive flow and peristalsis, and in a highly viscous environment. Thus, trypanosome migration likely relies on active and directional motion.

Trypanosome motility has been extensively analyzed from culture observations (Ralston et al., 2009). If the trypanosome flagellum can produce bidirectional waves, tip to base beating is observed most of the time in liquid medium, and this results in the cell traction (Branche et al., 2006). However, the natural environments of trypanosomes are not as liquid as the commonly used synthetic culture media. Consequently, despite important technical issues, analyses of trypanosome motion in their natural environments are now required.

T. brucei variant surface glycoproteins form a dense surface coat involved in antigenic variation. Their sorting by hydrodynamic forces is though to help trypanosomes to protect against complement-mediated immune destruction (Engstler et al., 2007). One could propose that cell orientation defects could render this mechanism inefficient. Nevertheless, the importance of forward swimming in vivo is far from a consensus, mostly because of the controversial RNAi phenotypes observed so far that are systematically lethal regardless of the targeted flagellum protein (Hill, 2010). In contrast, little or no growth deficit has been reported in motility-deficient procyclic trypanosomes in culture such as in DNAl1 ${ }^{\text {RNAi }}$ (Branche et al., 2006), PFR2 $2^{R N A i}$ (Bastin et al., 1998), Trypanin ${ }^{R N A i}$ (Hutchings et al., 2002) or $L C 1^{R N A i}$ mutants (Baron et al., 2007, Ralston et al., 2011). In agreement with these observations of monomorphic strains in vitro, the pleomorphic DNAI1 mutant parasites were still able to 
invade the posterior midgut and to proliferate. Moreover, despite having motility that is strongly impaired, they were able to reach the anterior midgut of the fly. Protozoan motions in low Reynold's number environments are not fully understood. In addition, the nature and physical properties of the viscous microenvironments imposed on trypanosomes in the tsetse midgut are likely to be frequently modified with the digestion process. This could influence parasite motion efficiency in a way that would favor, or at least not disfavor, the backward movements of DNAI1 mutants. Another, yet more unlikely, explanation is a possible cooperation between cells that could synchronize their movements or promote cooperation (Oberholzer et al., 2010). This would result in a progressive movement of DNAI1 mutants in the direction of the tsetse proventriculus. However, this migration was limited to the anterior region of the midgut in DNAl1 mutant trypomastigotes, suggesting that the conditions for parasite migration after this point could be different. From this region of the digestive tract, forward motility appeared to be crucial for parasite migration. Again, it could be hypothesized that the nature of the microenvironment (e.g. lower viscosity around the proventriculus), and/ or the reduction in parasites density (Oberle et al., 2010).

An unexpected modification in parasite development emerged in the DNAI1 mutant strain: the absence of epimastigote forms in the anterior midgut. Although the link between flagellum beating and the molecular processes involved in cytoskeleton remodeling and nucleus repositioning remain unclear (Sharma et al., 2008, Rotureau et al., 2011), this could reflect for the first time a direct involvement of motility in cell differentiation. As the parasite flagellum was proposed to act as a sensing antenna that could scan the micro-environment at the front of the cell (Oberholzer et al., 2011)(Subota et al., unpublished data), it could be proposed that an incorrect orientation of the flagellar tip during the migration, or the preponderance of base to tip waves, could prevent the proper reception of external stimuli and / or the subsequent intra-cellular signaling of information leading to the initiation of differentiation.

Our difficulties to fully knockout DNAl1 genes due to the presence of an extra copy nonetheless allowed us to produce mutant parasites with strong motility defects but yet 
viable. This reinforces the importance of the flagellar outer dynein arms in trypanosome biology as the DNAl1 mutant mesocyclic parasites were neither able to reach the foregut nor able to differentiate into epimastigote forms, two key steps of parasite development. The complementation of the phenotypes observed in DNAl1 mutants by the addition of a rescue copy of the DNAl1 gene confirms that both the absence of forward motility and the inability to differentiate into epimastigotes are specifically due to the reduction of the amount of DNAI1 in mutant parasites. In other words, forward motility is essential for the long mesocyclic trypomastigote parasites to assume their function of invading the tsetse foregut and paving the way for the parasite population to ultimately infect to tsetse salivary glands. In summation, this is the first demonstration that trypanosome swimming is essential for the completion of the parasite cycle in vivo. The fact that motility impairment could break the parasite cycle opens new approaches for the control of diseases caused by trypanosomatids. 


\section{Experimental procedures}

\section{Trypanosome cell lines and cultures}

All vectors were separately nucleofected (Lonza) in the pleomorphic strain Trypanosoma brucei brucei AnTat1.1 (Le Ray et al., 1977) at the freshly differentiated procyclic stage (<2 weeks). Procyclic trypanosomes were maintained in SDM79 medium (Brun et al., 1979) supplemented with $10 \%$ fetal bovine serum and $20 \mathrm{mM}$ glycerol. Antibiotic-resistant cells with the most pronounced motility phenotype or the highest EGFP signal were selected for sub-cloning by limiting dilution and named M1 to M6. Cell culture growth was monitored daily with a Z2 cell counter (Beckman Coulter).

\section{Generation of DNAl1 mutant and AB cell lines}

Two alleles of the DNAl1 gene (Tb11.02.2640) were successively replaced by two distinct antibiotic resistance cassettes. Constructs composed of the full BLA (399 bp) and NEO (867 bp) coding sequences flanked by the first $100 \mathrm{bp}$ upstream the start codon and downstream the stop codon of the DNAl1 coding sequence were chemically synthesized (GeneCust Europe, sequences in Figure S1). Inserts were amplified with the primers F1 and R1 selected to hybridize to the $100 \mathrm{bp}$ region immediately flanking the DNAl1 coding sequence that were used for recombination of the two resistance markers: CGAAGCAACCGTAGAGGACTCCGTAGT as forward primer F1 and TTGCCTCATTCATAATCCATCACAAAC as reverse primer R1. Inserts were integrated in the trypanosome genome via homologous recombination in the DNAl1 locus via two successive rounds of nucleofection (Lonza). Subclones of double mutant cells were transformed with a rescue copy of the DNA/1 gene tagged to EGFP and integrated in the PFR intergenic region of the trypanosome nuclear genome to be used as an add-back control strain $(A B)$. For this purpose, the full DNAl1 coding sequence $(1,968 \mathrm{bp})$ was cloned with Nhel and EcoRl at the 3' end of the EGFP coding sequence in the pPCPFR-EGFP vector (Absalon et al., 2008, Adhiambo et al., 2009). 


\section{Tsetse fly infection, maintenance and dissection}

Teneral males of Glossina morsitans morsitans from 24 to 96 hours post-eclosion were obtained from the UMR177 IRD-CIRAD, Campus International de Baillarguet, Montpellier, France. Tsetse flies were allowed to ingest parasites in culture medium during their first meal through a silicone membrane. Cultured procyclic trypanosomes were used at $5 \times 10^{6}$ cells per $\mathrm{ml}$ in SDM79 medium supplemented with $10 \%$ foetal bovine serum, $60 \mathrm{mM} \mathrm{N}$ acetylglucosamine (Peacock et al., 2006) and $2.5 \%(\mathrm{w} / \mathrm{v})$ bovine serum albumin (Kabayo et al., 1986). Tsetse flies were subsequently maintained in Roubaud cages at $27^{\circ} \mathrm{C}$ and $70 \%$ hygrometry and fed twice a week through a silicone membrane with fresh defibrinated sheep blood.

Flies were starved for at least 48 hours before being dissected from 26 to 34 days after ingestion of the infected meal. Whole tsetse alimentary tracts, from the distal part of the foregut to the rectum, were then dissected and arranged lengthways for assessment of parasite presence. Foregut and proventriculus were physically separated from the midgut in distinct PBS drops. Tissues were dilacerated and recovered parasites were treated for further experiments no more than 15 minutes after dissection.

\section{Motility analyses}

For motility analysis, two different tests were performed in three separate experiments: sedimentation assay and in silico tracking. Interfering with flagellum motility reduces cell motility resulting in cell sedimentation at the bottom of the culture flask. This can be monitored in sedimentation assays by measuring the optical density (OD) of cultures as previously described (Bastin et al., 1999, Branche et al., 2006). In addition, the 2D tracks characterizing cell motility were obtained by in silico tracking experiments. For each strain, 10-20 movies were recorded (200 frames, $50 \mathrm{~ms}$ of exposure). Samples were observed in culture medium maintained at $27{ }^{\circ} \mathrm{C}$ at $5 \times 10^{6}$ cells / ml under the $10 \mathrm{x}$ objective of an inverted DMI-4000B microscope (Leica) coupled to a Retiga-SRV camera (QImaging). Movies were converted with the MPEG Streamclip V.1.9b3 software (Squared 5) and 
analyzed with the medeaLAB CASA Tracking V.5.5 software (medea AV GmbH). For a more precise observation, movies of individual cells (200 frames, $100 \mathrm{~ms}$ of exposure) were also recorded. Samples from cultures or dissected organs were observed under a 100x NA 1.4 Plan Apo objective and processed as above. Movies were compiled in iMovie 9.0.4 (Apple).

\section{Immunofluorescence}

For immunofluorescence, parasites extracted from the anterior midgut were settled on poly-

-lysine coated slides and fixed in methanol at $-20^{\circ} \mathrm{C}$ for at least 5 minutes and re-hydrated in PBS for 10 minutes. Slides were stained with 4',6-diamidino-2-phenylindole (DAPI) for visualization of kinetoplast and nuclear DNA content, washed and mounted under cover slips with ProLong antifade reagent (Invitrogen). Samples were observed either with (i) a DMR nicroscope (Leica) and images were captured with a CoolSnap HQ camera (Roper Scientific), (ii) with a DMI4000 microscope (Leica) and images were acquired with a RetigaSRV camera (Q-Imaging).

\section{Electron microscopy}

For preparation of cytoskeletons, cells were treated with $1 \%$ Nonidet $\mathrm{P}-40$ at $4{ }^{\circ} \mathrm{C}$ in PBS for 10 minutes to strip the plasma membrane. Samples were washed twice in PBS, fixed, embedded and sectioned for transmission electron microscopy as described previously (Branche et al., 2006).

\section{PCR and RT-PCR}

Genomic DNA was extracted from each strain and purified using phenol / chloroform (Rotureau et al., 2005). PCR was performed according to the manufacturer recommendations with a GoTaq kit (Promega). Primers F2 and R2 were selected to hybridize $200 \mathrm{bp}$ upstream of the start codon and $200 \mathrm{bp}$ downstream of the stop codon of the DNAl1 coding sequence, in order to verify the correct insertions of the drug markers (Figure 1A): CCATCCTTCAAGTACACCATCAAT as forward primer F2 and GAGGATATATACACACACTTATTA as a reverse primer R2. 
Total RNAs were extracted from each strain and purified using TRIzol (Invitrogen). DNA was eliminated by DNase treatment (Qiagen) and RNA purity was confirmed by conventional PCR. After primer calibration and determination of optimal conditions, semi-quantitative RTPCR was performed according to the manufacturer recommendations with a SuperScript One-step RT-PCR Platinum-Taq kit (Invitrogen). Primers were selected to amplify short regions in the coding sequences of the following genes: GAATCCGTTCCATCCCGATGTCTT (forward) and TCACGCCTGCCCCAGGA (reverse) targeting a $470 \mathrm{bp}$ sequence of DNAl1, and ATGGGTGGCAGGACT (forward) and GCCCACGGTTTGGTC (reverse) targeting the first 105 bp of the FLA1 gene as control. In parallel to RT-PCR targeting DNAl1, the same samples were processed for RT-PCR targeting FLA1 and for direct PCR targeting DNAl1 as positive and negative controls respectively. Internal negative controls with $\mathrm{H}_{2} \mathrm{O}$ for RT-PCR and positive control with WT genomic DNA for PCR were also performed.

Statistical analyzes

Statistical analyses were performed in Excel or with the KaleidaGraph V.4.0 software

(Synergy Software). Infection rates were plotted as mean \pm SD. One-way ANOVA tests, with

intergroup comparisons by Tukey ad-hoc post-tests with $\alpha=0.05$, were performed and

significant results were indicated with * $p<0.05$ (Fig. 3A). 


\section{Acknowledgements}

We thank the UMR177 IRD-CIRAD team headed by G. Cuny (Montpellier, France) for providing tsetse flies. We are grateful to J. Van Den Abbeele for providing the trypanosome AnTat 1.1 cell line. This work was funded by the Institut Pasteur and the CNRS. This study has received funding from the French National Agency for Research (grant ANR MIE08-027 SENSOTRYPA), and from the French Government's Investissement d'Avenir program, Laboratoire d'Excellence "Integrative Biology of Emerging Infectious Diseases" (grant $\mathrm{n}^{\circ}$ ANR-10-LABX-62-IBEID). CPO is funded by a DIM lle de France post-doctoral fellowship, and $\mathrm{DH}$ by a doctoral FRM fellowship and a ED387 grant from the French Ministry of Research. The authors declare no competing financial interests. 


\section{Figure Legends}

\section{Figure 1: DNAl1 mutants loose part of their outer dynein arms.}

(A) The two alleles of the DNAl1 gene were successively replaced via homologous recombination by the blasticidin (BLA) and neomycin (NEO-G418) resistance marker coding sequences, flanked by the DNAl1 flanking sequences (black bars), to produce the DNAl1 mutant strain. The relative positions of the PCR primer pairs used for amplification of the inserts (F1 and R1 in orange) and integration control (F2 and R2 in red) are indicated. (B) PCR with the primers F2 and R2, designed to hybridize outside the first 100 bp of the DNAl1 flanking sequences used for recombination, was performed on $100 \mathrm{ng}$ of genomic DNA from WT, DNAl1 mutant subclones 1 to 6 (M1 to M6) and cells transformed with only one insert or the other (B1 and B2 for blasticidin-resistant subclones, and G1 and G2 for neomycin/G418resistant subclones). The expected resistance marker cassettes were detected in all the different clones for each construct (596 bp for BLA and 1,067 bp for NEO/G418). In addition, a band detected in all mutant types and clones, whatever the order of the transformation process, and with the same size as the full DNAl1 WT gene $(2,165 \mathrm{bp})$, was sequenced and proved to correspond to an extra-copy of the gene. (C) RT-PCR targeting the DNAl1 coding sequence was performed on total RNA extracts from WT parasites, simple (B2) and double (M1 and M2) DNAl1 mutant subclones, as well as AB cells. In parallel, the same samples were processed for RT-PCR targeting FLA1 mRNA and for direct PCR targeting FLA1 DNA as positive and negative controls respectively. Internal negative controls with $\mathrm{H}_{2} \mathrm{O}$ for RTPCR and positive control with genomic DNA for PCR are shown in the last column (+/-).

Cell cytoskeletons were extracted by detergent treatment and processed for transmission EM. Flagella cross-sections were analyzed for each strain. Asterisks indicate microtubule doublets where outer dynein arms are missing. Scale bar represents $200 \mathrm{~nm}$. (E) Numbers of missing outer dynein arms were quantified in transmission EM pictures of detergent-treated flagellum cross-sections and plotted as percentage of the total observed cross-sections for each strain. 
Figure 2. DNAl1 mutants present impaired motility but are still viable.

(A) Representative pictures $(n>12)$ of 200 individual tracks from WT, DNAl1 mutant subclone 2 and $A B$ cells showing trypanosome movements analyzed over 20 s by in silico tracking. (B) Representative results from a sedimentation assay $(n=3)$ showing the variations of optical densities measured over 8 hours that directly reflect the evolution of the turbidity in the culture medium. Immotile DNA/1 mutant parasites (M2) rapidly accumulated at the bottom of culture flasks in liquid medium in contrast to WT and $A B$ cells that were homogeneously distributed. (C) Representative in vitro growth curve $(n=3)$ showing cell densities of WT, DNAl1 mutants (M2) and AB cells over 96 hours.

Figure 3. DNAl1 mutant trypomastigotes cannot reach the proventriculus nor differentiate into epimastigotes.

Flies were fed with WT trypanosomes, DNAI1 mutant subclones 1 and 2 (M1 and M2) or AB cells, and dissected from 26 to 34 days after. Whole tsetse alimentary tracts were arranged lengthways for assessment of parasite presence. PMG: posterior midgut; AMG: anterior midgut; FG: foregut; SG: salivary glands. (A) Infection rates were plotted as mean \pm SD according to the region of the digestive tract. Total numbers of dissected flies from at least three separate experiments are indicated. One-way ANOVA tests, with intergroup comparisons by Tukey ad-hoc post-tests with $\alpha=0.05$, were performed and significant results were indicated with * $p<0.05$. (B) Parasites extracted from the AMG of infected flies were fixed in methanol and stained with DAPI. Arrows indicate epimastigote forms in representative fields for $\mathrm{WT}$ and $\mathrm{AB}$ infections. Scale bar represents $10 \mu \mathrm{m}$. (C) Parasites were typed according to their morphology and DNA staining pattern (relative positions of the kinetoplasts and nuclei). Cell populations were quantified and plotted by stage as percentage of the total number of parasites for each strain. Total numbers of cells randomly observed from at least three distinct experiments are given for each strain. PC: procyclic 
trypomastigote; MS: mesocyclic trypomastigote; DE: long dividing epimastigote; LE: long epimastigote; SE: short epimastigote. 


\section{Supplemental information}

Movie S1: DNAl1 mutant motility defects in vitro.

Movies of individual WT, DNAI1 mutant M2 and AB trypanosomes observed in liquid culture medium under an $\times 100$ objective.

Movie S2: DNAI1 mutant motility defects in vivo.

Movies of trypanosomes extracted from the anterior midgut of tsetse flies fed 26 days before with WT, DNAI1 mutant M2 and AB trypanosomes and observed under a 40x objective. 


\section{References}

Absalon, S., Blisnick, T., Kohl, L., Toutirais, G., Dore, G., Julkowska, D., et al. (2008). Intraflagellar Transport and Functional Analysis of Genes Required for Flagellum Formation in Trypanosomes. Mol Biol Cell 19, 929-944.

Adhiambo, C., Blisnick, T., Toutirais, G., Delannoy, E. and Bastin, P. (2009). A novel function for the atypical small $G$ protein Rab-like 5 in the assembly of the trypanosome flagellum. J Cell Sci 122, 834-841.

Baron, D.M., Kabututu, Z.P. and Hill, K.L. (2007). Stuck in reverse: loss of LC1 in Trypanosoma brucei disrupts outer dynein arms and leads to reverse flagellar beat and backward movement. J Cell Sci 120, 1513-1520.

Bastin, P., Pullen, T.J., Sherwin, T. and Gull, K. (1999). Protein transport and flagellum assembly dynamics revealed by analysis of the paralysed trypanosome mutant snl-1. J Cell Sci 112 ( Pt 21), 3769-3777.

Bastin, P., Sherwin, T. and Gull, K. (1998). Paraflagellar rod is vital for trypanosome motility. Nature 391, 548.

Branche, C., Kohl, L., Toutirais, G., Buisson, J., Cosson, J. and Bastin, P. (2006). Conserved and specific functions of axoneme components in trypanosome motility. J Cell Sci $119,3443-3455$.

Brun, R., Blum, J., Chappuis, F. and Burri, C. (2009). Human African trypanosomiasis. Lancet 375, 148-159.

Brun, R. and Schonenberger (1979). Cultivation and in vitro cloning or procyclic culture forms of Trypanosoma brucei in a semi-defined medium. Short communication. Acta Trop $36,289-292$.

Chappuis, F., Lima, M.A., Flevaud, L. and Ritmeijer, K. (2010). Human African trypanosomiasis in areas without surveillance. Emerg Infect Dis 16, 354-356.

Engstler, M., Pfohl, T., Herminghaus, S., Boshart, M., Wiegertjes, G., Heddergott, N. and Overath, P. (2007). Hydrodynamic flow-mediated protein sorting on the cell surface of trypanosomes. Cell 131, 505-515. 
Griffiths, S., Portman, N., Taylor, P.R., Gordon, S., Ginger, M.L. and Gull, K. (2007). RNA interference mutant induction in vivo demonstrates the essential nature of trypanosome flagellar function during mammalian infection. Eukaryot Cell 6, 12481250.

Heintzelman, M.B. (2006). Cellular and molecular mechanics of gliding locomotion in eukaryotes. Int Rev Cytol 251, 79-129.

Hill, K.L. (2010). Parasites in motion: flagellum-driven cell motility in African trypanosomes. Curr Opin Microbiol 13, 459-465.

Hillyer, J.F., Barreau, C. and Vernick, K.D. (2007). Efficiency of salivary gland invasion by malaria sporozoites is controlled by rapid sporozoite destruction in the mosquito haemocoel. Int J Parasitol 37, 673-681.

Hutchings, N.R., Donelson, J.E. and Hill, K.L. (2002). Trypanin is a cytoskeletal linker protein and is required for cell motility in African trypanosomes. J Cell Biol 156, 867-877.

Kabayo, J.P., DeLoach, J.R., Spates, G.E., Holman, G.M. and Kapatsa, G.M. (1986). Studies on the biochemical basis of the nutritional quality of tsetse fly diets. Comp Biochem Physiol A Comp Physiol 83, 133-139.

Kaye, P. and Scott, P. (2011). Leishmaniasis: complexity at the host-pathogen interface. Nat Rev Microbiol 9, 604-615.

Le Ray, D., Barry, J.D., Easton, C. and Vickerman, K. (1977). First tsetse fly transmission of the "AnTat" serodeme of Trypanosoma brucei. Ann Soc Belg Med Trop 57, 369-381.

Oberholzer, M., Langousis, G., Nguyen, H.T., Saada, E.A., Shimogawa, M.M., Jonsson, Z.O., et al. (2011). Independent analysis of the flagellum surface and matrix proteomes provides insight into flagellum signaling in mammalian-infectious Trypanosoma brucei. Molecular \& cellular proteomics : MCP 10, M111 010538.

Oberholzer, M., Lopez, M.A., McLelland, B.T. and Hill, K.L. (2010). Social motility in african trypanosomes. PLoS Pathog 6, e1000739. 
Oberle, M., Balmer, O., Brun, R. and Roditi, I. (2010). Bottlenecks and the maintenance of minor genotypes during the life cycle of Trypanosoma brucei. PLoS Pathog 6, e1001023.

Peacock, L., Ferris, V., Bailey, M. and Gibson, W. (2006). Multiple effects of the lectininhibitory sugars D-glucosamine and $\mathrm{N}$-acetyl-glucosamine on tsetse-trypanosome interactions. Parasitology 132, 651-658.

Peacock, L., Ferris, V., Bailey, M. and Gibson, W. (2007). Dynamics of infection and competition between two strains of Trypanosoma brucei brucei in the tsetse fly observed using fluorescent markers. Kinetoplastid Biol Dis 6, 4.

Ralston, K.S., Kabututu, Z.P., Melehani, J.H., Oberholzer, M. and Hill, K.L. (2009). The Trypanosoma brucei flagellum: moving parasites in new directions. Annu Rev Microbiol 63, 335-362.

Ralston, K.S., Kisalu, N.K. and Hill, K.L. (2011). Structure-function analysis of dynein light chain 1 identifies viable motility mutants in bloodstream-form Trypanosoma brucei. Eukaryot Cell 10, 884-894.

Robinson, D.R. and Gull, K. (1991). Basal body movements as a mechanism for mitochondrial genome segregation in the trypanosome cell cycle. Nature 352, 731733.

Rotureau, B., Gego, A. and Carme, B. (2005). Trypanosomatid protozoa: a simplified DNA isolation procedure. Exp Parasitol 111, 207-209.

Rotureau, B., Subota, I. and Bastin, P. (2011). Molecular bases of cytoskeleton plasticity during the Trypanosoma brucei parasite cycle. Cell Microbiol 13, 705-716.

Rotureau, B., Subota, I., Buisson, J. and Bastin, P. (2012). A new asymmetric division contributes to the continuous production of infective trypanosomes in the tsetse fly. Development 139, 1842-1850.

Sharma, R., Gluenz, E., Peacock, L., Gibson, W., Gull, K. and Carrington, M. (2009). The heart of darkness: growth and form of Trypanosoma brucei in the tsetse fly. Trends Parasitol 25, 517-524. 
Sharma, R., Peacock, L., Gluenz, E., Gull, K., Gibson, W. and Carrington, M. (2008). Asymmetric cell division as a route to reduction in cell length and change in cell morphology in trypanosomes. Protist 159, 137-151.

Simarro, P.P., Jannin, J. and Cattand, P. (2008). Eliminating human African trypanosomiasis: where do we stand and what comes next? PLoS Med 5, e55.

Subota, I., Rotureau, B., Blisnick, T., Ngwabyt, S., Durand-Dubief, M., Engstler, M. and Bastin, P. (2011). ALBA proteins are stage regulated during trypanosome development in the tsetse fly and participate in differentiation. Mol Biol Cell 22, 42054219.

Tavares, P., Sansonetti, P. and Guillen, N. (2000). Cell polarization and adhesion in a motile pathogenic protozoan: role and fate of the Entamoeba histolytica Gal/GalNAc lectin. Microbes Infect 2, 643-649.

Van Den Abbeele, J., Claes, Y., van Bockstaele, D., Le Ray, D. and Coosemans, M. (1999). Trypanosoma brucei spp. development in the tsetse fly: characterization of the postmesocyclic stages in the foregut and proboscis. Parasitology 118, 469-478.

Vickerman, K. (1985). Developmental cycles and biology of pathogenic trypanosomes. $\mathrm{Br}$ Med Bull 41, 105-114. 
Pade 27 of 29

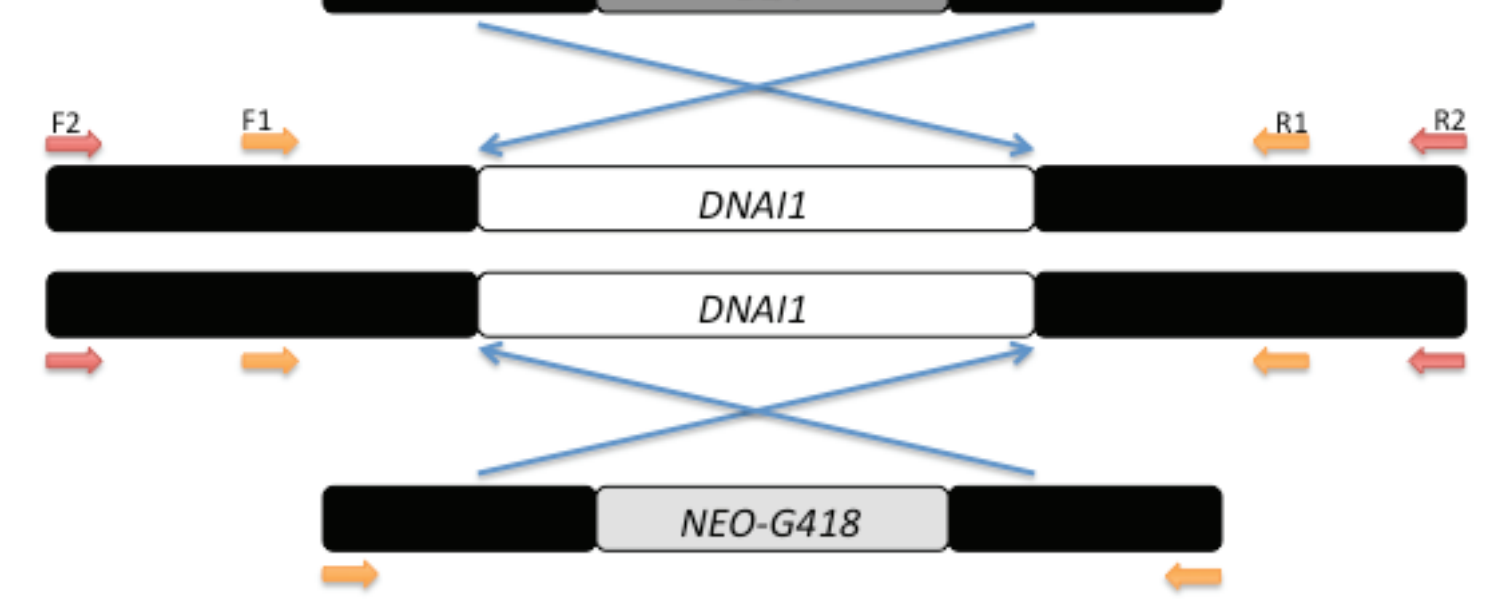

16

$17 \mathbf{B}$

20DNAl1

21

22NEO-G418

${ }_{24}^{23}$ Blasticidin

24

${ }_{28}^{27} \mathrm{C}$

RT-PCR DNA11

RT-PCR FLA1

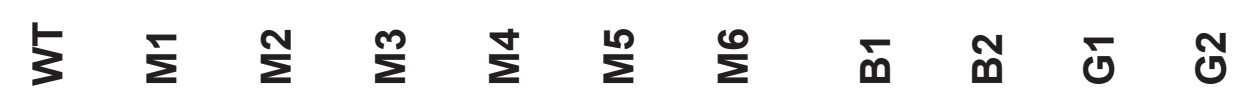

- $-0-0-0$

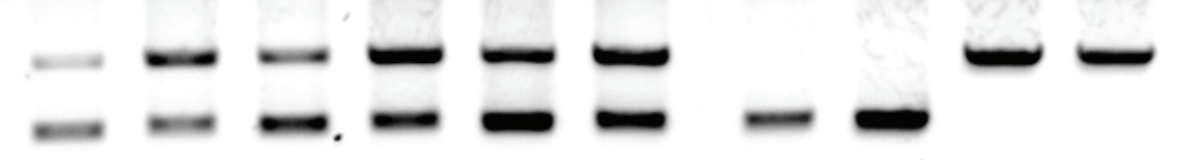

PCR DNAl1
WT M2

AB
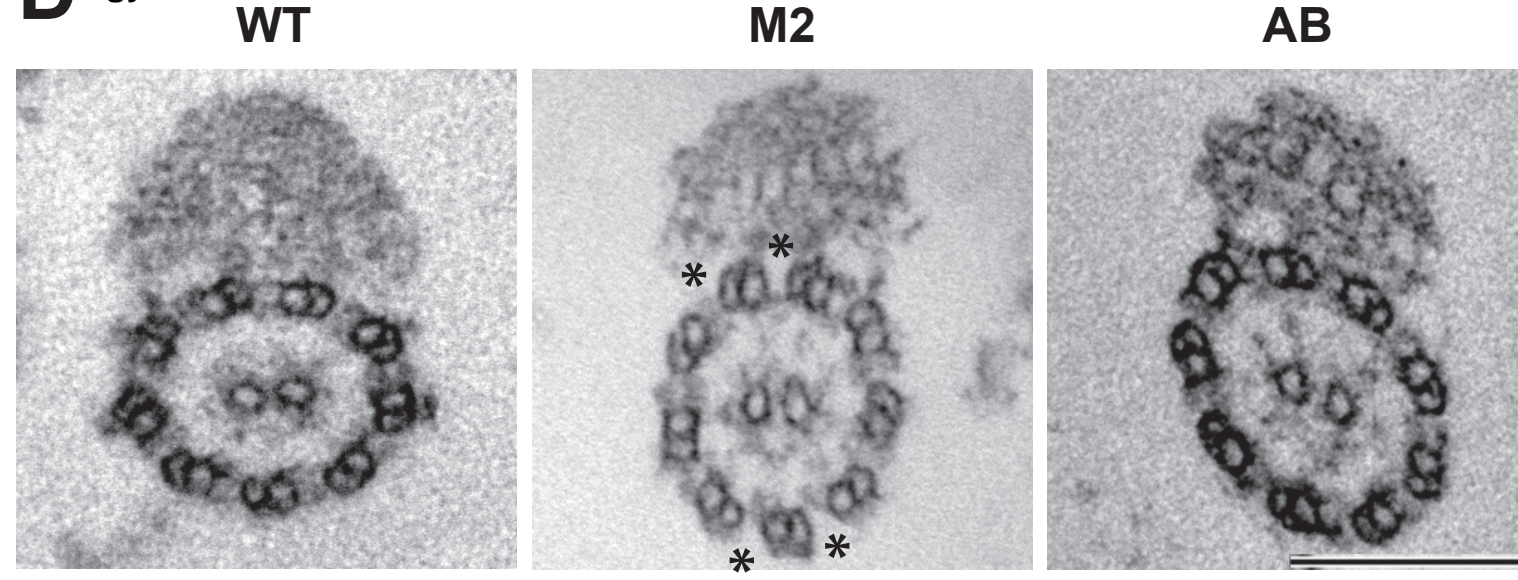

E

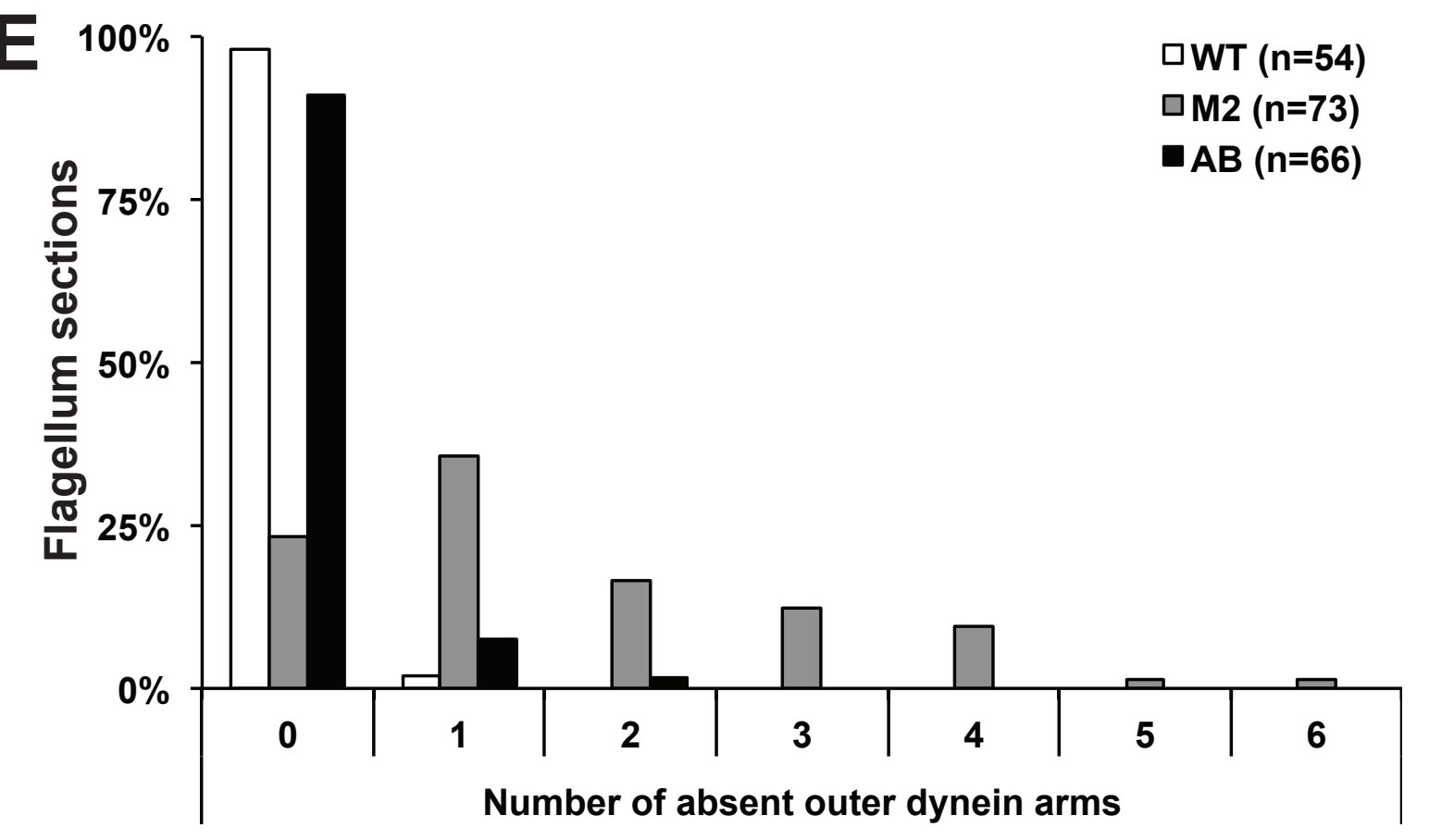

Figure 1 
A

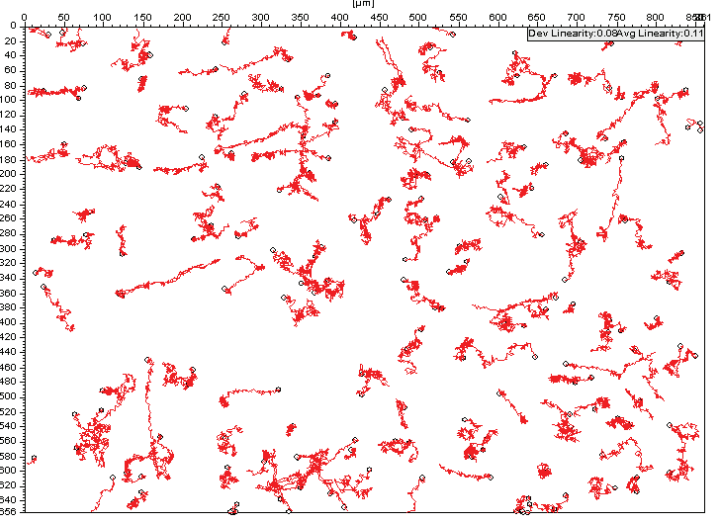

12

12
13

14
B oseztolar Microbiology

Page 28 of 29
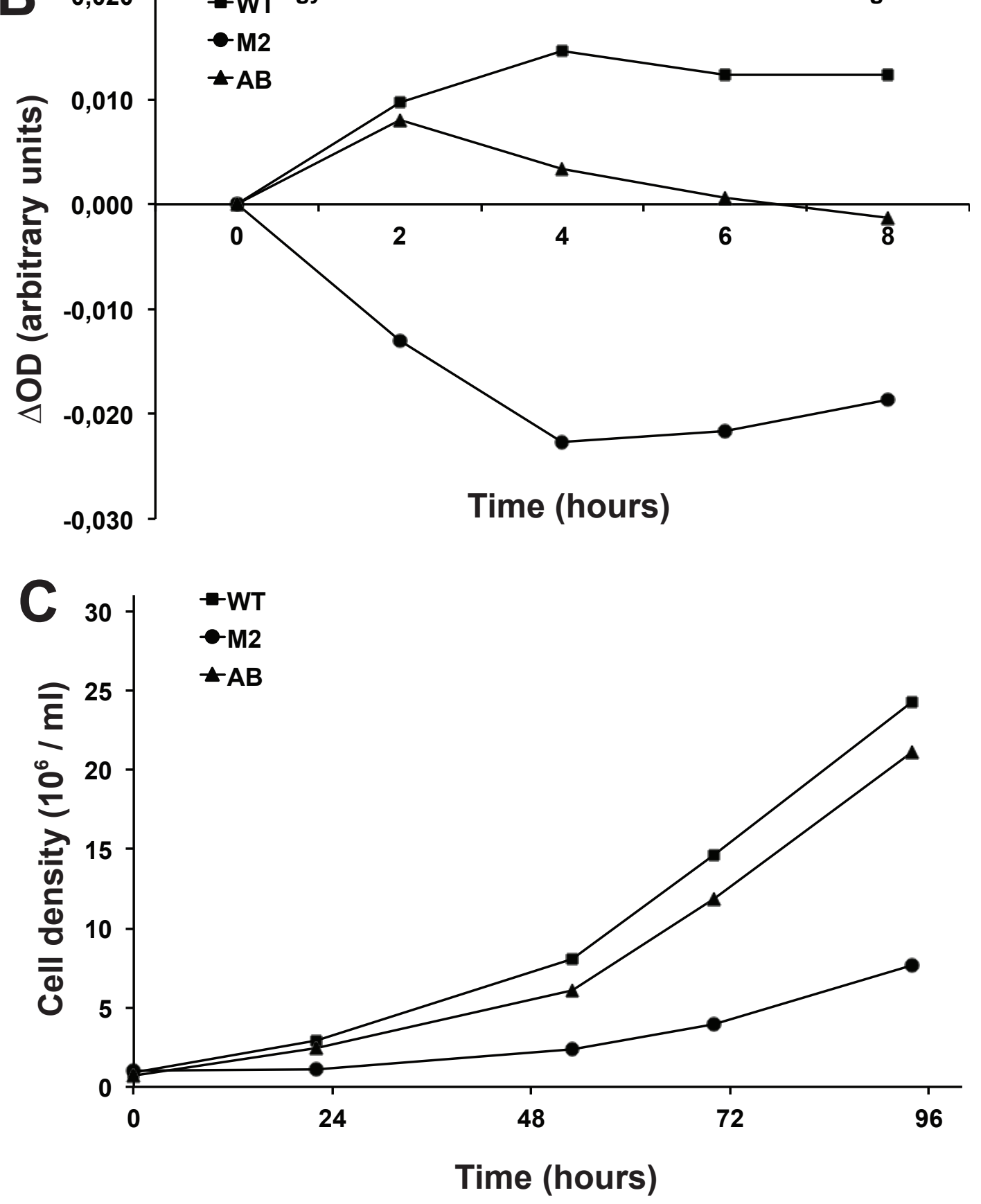

Figure 2 


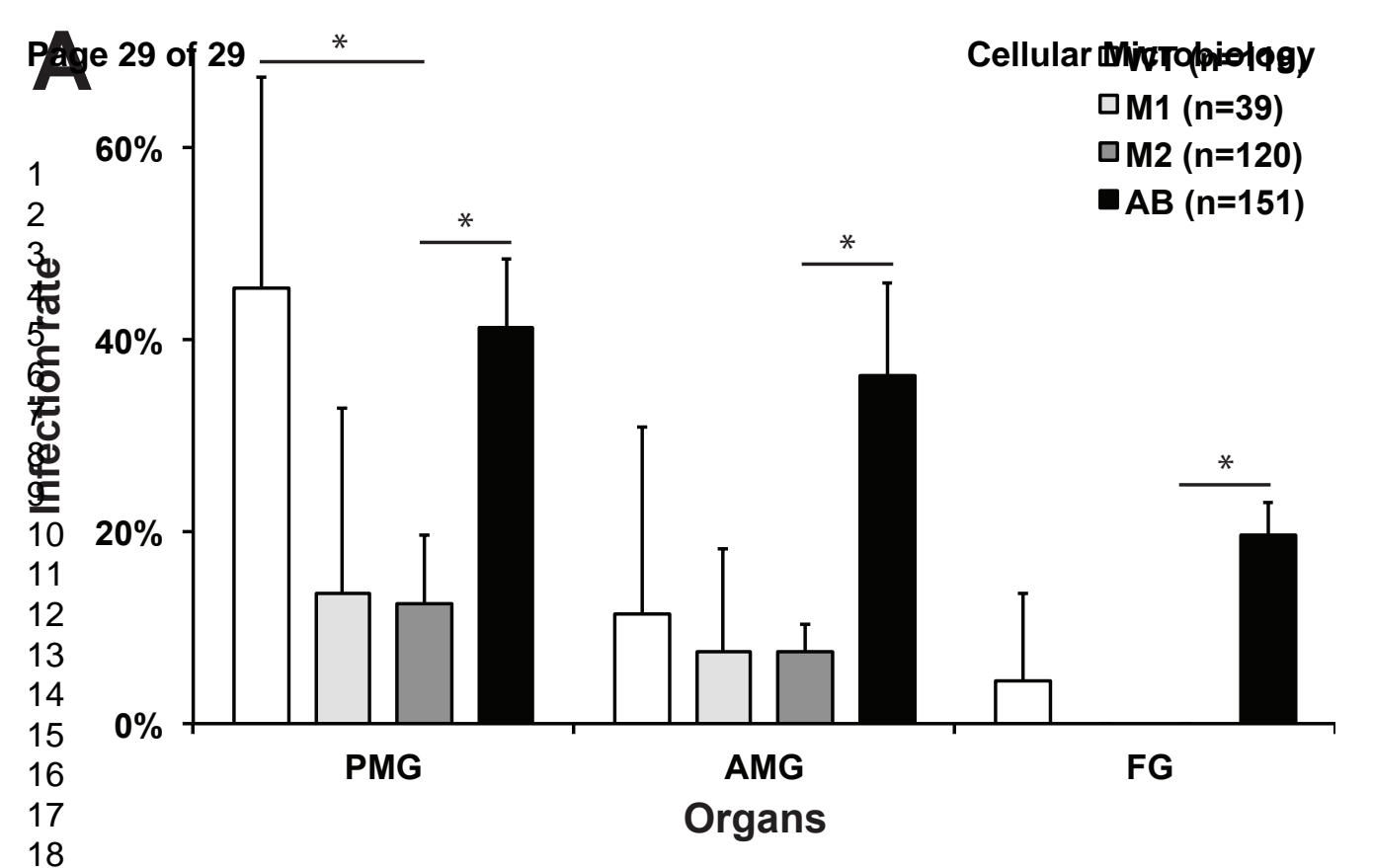

19
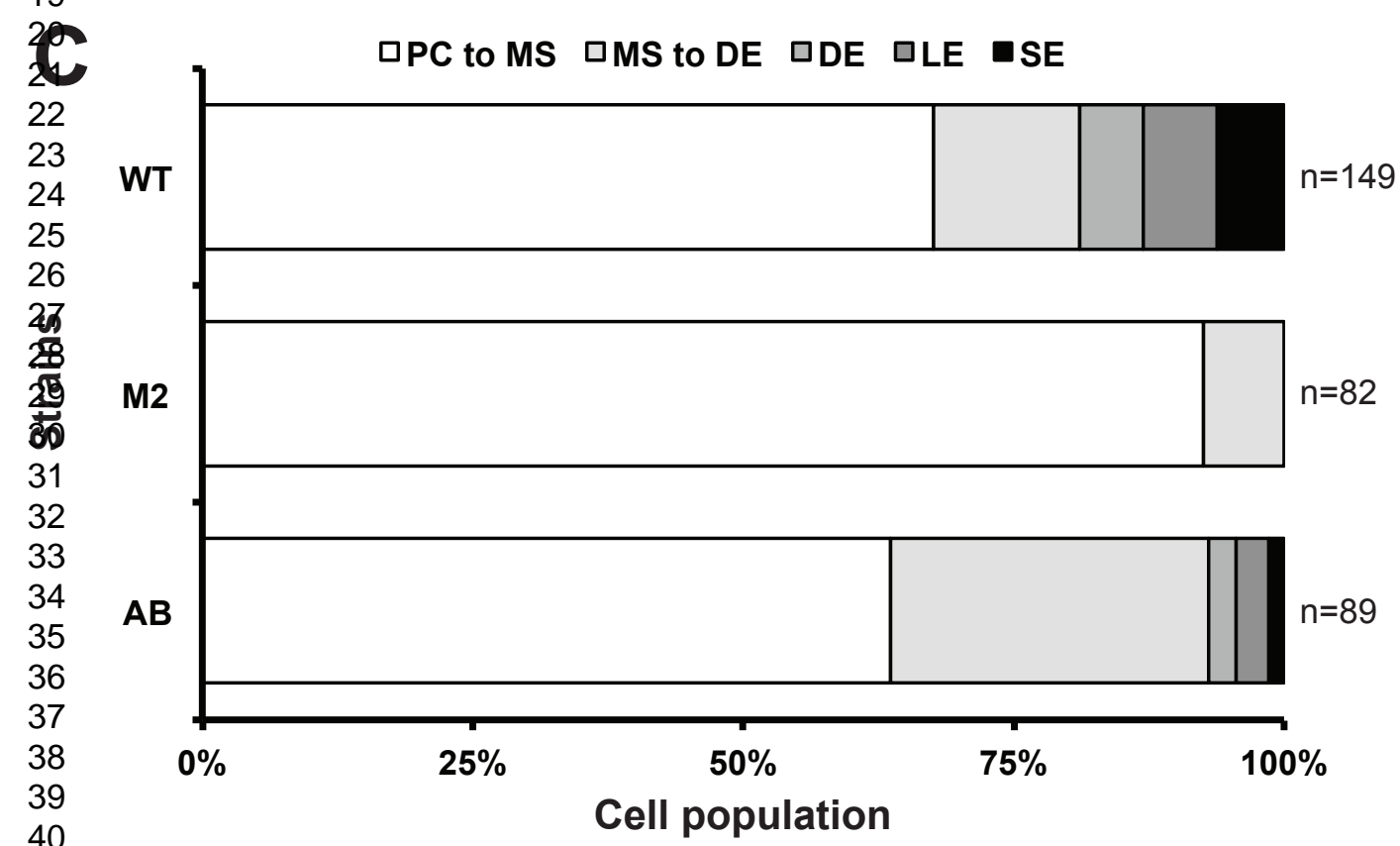

B
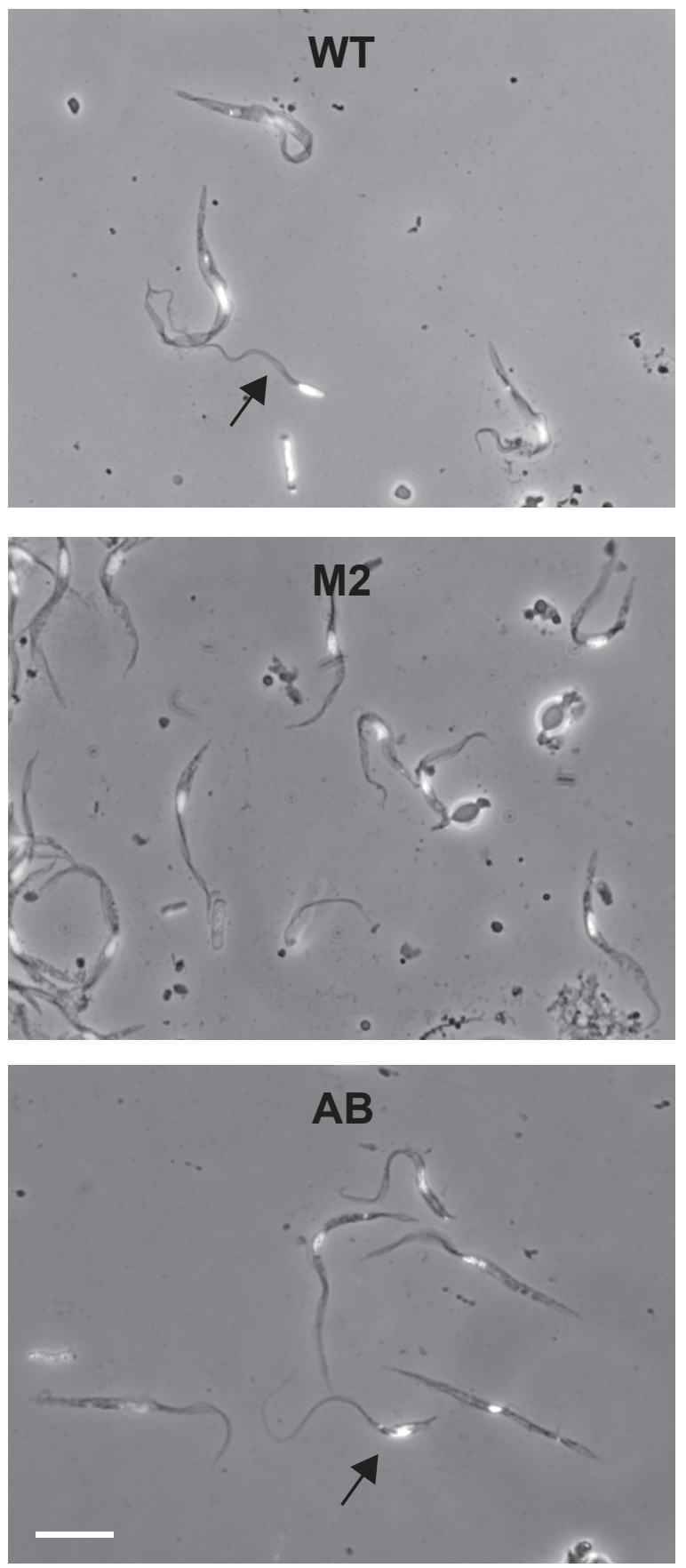

Figure 3 